\title{
WHO guidelines on ethical public health surveillance
}

Cite as: CMAJ 2017 July 24;189:E977. doi: 10.1503/cmaj.1095453

Posted on cmajnews.com on July 6, 2017.

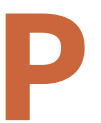

ublic health surveillance is "the foundation for initiatives to promote human well-being at the population level," according to the World Health Organization (WHO). But there is potential for groups and individuals to be harmed when information about their health is collected and shared. To address this problem, the WHO has released guidelines on how to conduct public health surveillance ethically.

"These WHO guidelines are bold and comprehensive," said Nancy Ondrusek, the manager of research and ethics for Public Health Ontario. "They identify an ethical imperative to conduct surveillance, while at the same time specifying appropriate safeguards to reduce the potential harms that might arise if surveillance is implemented poorly or misused. I am also pleased to see that the guidelines tackled potentially thorny issues regarding collection without informed consent and use of surveillance data for research."

The WHO defines surveillance as "the continuous, systematic collection, analysis and interpretation of health-related data needed for the planning, implementation, and evaluation of public health practice." These data are used to map the spread of disease, find patterns and causes during outbreaks, and to plan interventions during epidemics. Surveillance data can also provide evidence to drive health policy, lead to better understanding of noncommunicable diseases, and identify "pockets of suffering" that are disproportionately affected by a particular disease.

"Public health surveillance is of critical importance for the function of national health systems and for global health," said Dr. Ross Upshur, former director of the Joint Centre for Bioethics at the University of Toronto and a contributor to the WHO guidelines. "However, the collection of personal

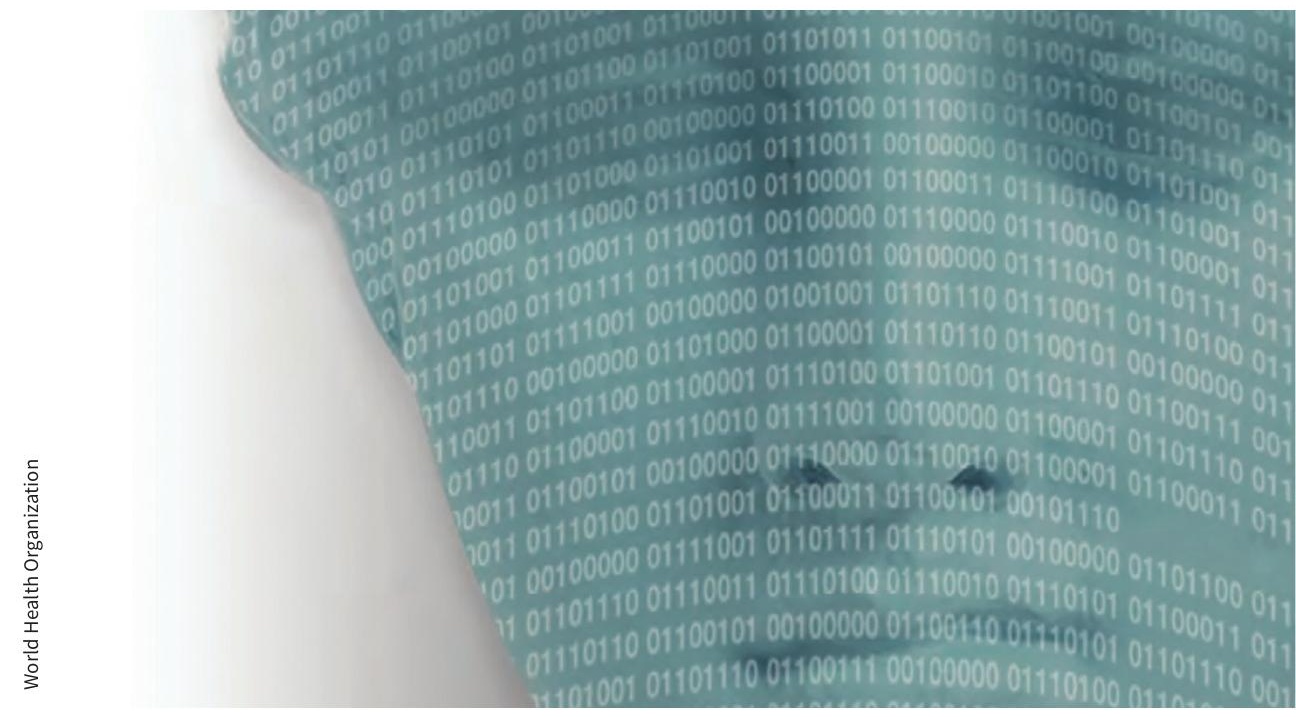

The goal of public health surveillance is to collect data to achieve public health goals without harming groups or individuals.

data for the benefit of public health raises important ethical questions, particularly about balancing confidentiality and privacy, governance, informed consent, et cetera, for which there existed no consistent guidance."

The collection of population-level health data can lead to mandatory quarantines, isolation of groups and individuals, and seizure of property. There is potential for intrusions of privacy and discrimination. Data that associate high HIV rates with particular communities, for example, can lead to stigmatization of members of those communities. Though the goal is to advance public health goals without harming individuals, people have experienced legal, social, physical and psychological harm after the public disclosure of health data.

"This document is the first document to provide health systems globally to address these issues and serves as a guide to integrate important ethical considerations into the design and implementation of their surveillance systems," said Upshur.
Some of the factors considered by the WHO in the development of the guidelines include respect for individuals, good governance, equity and the common good. There are 17 guidelines in all, addressing topics such as developing effective mechanisms for ethical surveillance, ensuring data are secure and collected only for legitimate health purposes, identifying and minimizing potential harms, assisting countries without the resources to conduct surveillance on their own, and sharing data with other nations and international health agencies.

"Individual organizations or jurisdictions will be able to look to these international guidelines when setting their own policies and procedures for surveillance," said Ondrusek. "Moreover, by adopting a common set of guidelines, countries can share data knowing it was collected and will be used according to appropriate ethical and scientific standards."

Roger Collier, CMAJ 